\title{
Cardiovascular phenotype and prognosis of patients with heart failure induced by cancer therapy
}

\author{
Wilson Nadruz, ${ }_{1}^{1,2}$ Erin West, ${ }_{1}^{1}$ Morten Sengeløv, ${ }_{1}^{1}$ Gabriela L Grove, ${ }_{1}^{1}$ Mário Santos, ${ }_{1}^{3}$ \\ John D Groarke, ${ }^{1}$ Daniel E Forman, ${ }^{4}$ Brian Claggett, ${ }^{1}$ Hicham Skali, ${ }^{1}$ Anju Nohria, ${ }^{1}$ \\ Amil M Shah ${ }^{1}$
}

\begin{abstract}
- Additional material is published online only. To view please visit the journal online (http://dx.doi.org/10.1136/ heartjnl-2018-313234).

${ }^{1}$ Division of Cardiovascular Medicine, Brigham and Women's Hospital, Boston, Massachusetts, USA ${ }^{2}$ Department of Internal Medicine, University of Campinas, Campinas, Brazil ${ }^{3}$ Faculty of Medicine, University of Porto, Porto, Portugal ${ }^{4}$ Department of Cardiology, University of Pittsburgh Medical Center and VA Pittsburgh Healthcare System, Pittsburgh, Pennsylvania, USA
\end{abstract}

\section{Correspondence to}

Dr Amil M Shah, Division of Cardiovascular Medicine, Brigham and Women's Hospital, Boston, MA 02445, USA; ashah11@rics.bwh.harvard.edu

Received 1 March 2018 Revised 10 April 2018 Accepted 23 April 2018 Published Online First 15 May 2018

\section{Linked}

- http://dx.doi.org/10.1136/ heartjnl-2018-313493

Check for updates

To cite: Nadruz W, West $\mathrm{E}$, Sengeløv $\mathrm{M}$, et al. Heart 2019;105:34-41.

\section{ABSTRACT \\ Objective This study compared the clinical}

features, cardiac structure and function evaluated by echocardiography, cardiopulmonary response to exercise and long-term clinical outcomes between patients with heart failure (HF) induced by cancer therapy (CTHF) and heart failure not induced by cancer therapy (NCTHF). Methods We evaluated 75 patients with CTHF and 894 with NCTHF who underwent clinically indicated cardiopulmonary exercise testing, and followed these individuals for a median of 4.5 (3.0-5.8) years, during which 187 deaths and 256 composite events (death, heart transplantation and left ventricular (LV) assistant device implantation) occurred.

Results Compared with NCTHF, patients with CTHF were younger, with lower prevalence of cardiovascular comorbidities, higher LV ejection fraction (LVEF), but similar global longitudinal strain. LV diastolic function (higher E/e' ratio) and compliance (higher end-diastolic pressure/LV end-diastolic volume index ratio) were worse in CTHF and were both associated with adverse outcomes. Despite a favourable clinical profile, peak $\mathrm{VO}_{2}$ and $\mathrm{VE} / \mathrm{VCO}_{2}$ slope were similarly impaired in CTHF and NCTHF. In multivariable Cox regression analysis including clinical characteristics, cardiopulmonary exercise testing variables and LVEF, CTHF was associated with a significantly higher risk of death (HR 2.64; $95 \% \mathrm{Cl}$ 1.53 to 4.55; $p=0.001)$ and composite events (HR 1.79; $95 \% \mathrm{Cl} 1.10$ to $2.91 ; p=0.019$ ) compared with NCTHF.

Conclusions CTHF is characterised by a distinct clinical profile, better LVEF but worse LV diastolic properties, and similarly impaired global longitudinal strain, functional capacity and ventilatory efficiency. Accounting for differences in clinical characteristics, CTHF was associated with worse long-term prognosis than NCTHF.

\section{INTRODUCTION}

Therapeutic advances have significantly improved cancer survival, with a concomitant increase in the prevalence of associated cardiotoxicity. ${ }^{1}$ The overall incidence of heart failure (HF) induced by cancer therapy (CTHF) depends on multiple factors including type of cancer therapies, duration of therapy and interval from treatment, but has been reported to be as high as $10 \%$ in cancer survivors, progressing to end-stage $\mathrm{HF}$ in $0.5 \%$ to $2.5 \% .^{12}$ CTHF may be caused by several chemotherapy agents, with anthracyclines being the most common offenders. ${ }^{3}{ }^{4}$ Radiation therapy to the chest is also a significant risk factor for HF, independent of adjuvant chemotherapy. ${ }^{5}$

Among patients with HF, there is limited information regarding the phenotype and prognosis of CTHF. Previous studies suggest that, compared with patients with HF not induced by cancer therapy (NCTHF), patients with CTHF are younger, more frequently women and have a lower prevalence of cardiovascular comorbidities. ${ }^{67}$ However, little data are available regarding cardiac structure and function, functional capacity and the haemodynamic response to exercise in CTHF relative to NCTHF, while data on the long-term prognosis of CTHF are conflicting. Prior studies have demonstrated higher, ${ }^{8}$ similar ${ }^{6910}$ or lower ${ }^{11}$ risk of death compared with NCTHF. Therefore, this study compared the clinical features, echocardiographic characteristics, cardiopulmonary response to exercise and long-term clinical outcomes in patients with CTHF and NCTHF.

\section{METHODS}

\section{Study population}

The present study included 973 consecutive patients referred for cardiopulmonary exercise testing (CPET) between July 2007 and December 2012 at the Brigham and Women's Hospital for an indication of HF or cardiomyopathy, as described previously. ${ }^{12}$ The majority of these patients were referred from our institution's advanced HF service where they were most commonly followed for severe HF for possible heart transplantation candidacy, persistent HF symptoms despite optimal medical therapy, or diagnostic evaluation. Participants with missing left ventricular (LV) ejection fraction (LVEF) data at baseline $(n=4)$ were excluded, resulting in 969 subjects for the current analysis. The study complies with the Declaration of Helsinki and was approved by the Partners Human Research Committee, which waived the informed consent requirement.

\section{Classification of patients with HF}

Among the study sample of 969 patients, 75 had CTHF while 894 patients had NCTHF. Previous malignancies in patients with CTHF included lymphoma (39\%), breast cancer (25\%), leukaemia (20\%), sarcoma (11\%), neuroblastoma (3\%), Wilm's tumour (1\%) and spindle cell carcinoma (1\%). Among patients with CTFH, 89\% and 44\% underwent treatment with anthracyclines and chest radiotherapy, respectively. The median interval 
between cancer therapy and HF diagnosis was $10(3,18)$ years, and the median interval between cancer therapy and CPET was $18(9,29)$ years.

\section{Clinical variables definition}

Patient demographics, blood pressure, heart rate, body mass index, medications and presence of pacemaker, implantable cardioverter defibrillator or cardiac resynchronisation therapy were collected at the time of CPET. Further clinical characteristics (New York Heart Association Classification (NYHA) and comorbidities) and laboratory values (creatinine and haemoglobin) closest to the CPET dates were obtained from chart review. Antiarrhythmic medications included amiodarone and digoxin. Glomerular filtration rate was estimated by the CKD-EPI formula. ${ }^{13}$ Chronic kidney disease was defined as estimated glomerular filtration rate $<60 \mathrm{~mL} / \mathrm{min} / 1.73 \mathrm{~m}^{2}$. Anaemia was defined as haemoglobin $<13 \mathrm{~g} / \mathrm{dL}$ in men and $<12 \mathrm{~g} / \mathrm{dL}$ in women. ACE inhibitors and angiotensin receptor blockers were coded as a single variable, as were implantable cardioverter defibrillator and cardiac resynchronisation therapy. In all studied patients, LVEF was abstracted from clinical reports of the echocardiography examination at the Brigham and Women's Hospital that was most contemporary to the CPET date. Patients with LVEF $\geq 40 \%$ (by clinical read) with a previously documented LVEF $<40 \%$ (by clinical read) were considered as having recovered LVEF, as previously described. ${ }^{12}$

\section{Echocardiography}

Cardiac structure and function were assessed from echocardiograms performed at the Brigham and Women's Hospital that were most proximate to the CPET. Quantitative echocardiographic data were available in $71(95 \%)$ patients with CTHF and 834 (93\%) with NCTHF. Quantitative analysis on digitally acquired echocardiographic studies was performed by a single expert technician who was blinded to clinical characteristics or HF aetiology. Assessment of LV and right ventricular structure and function, and left atrial structure were performed as previously described. ${ }^{14} \mathrm{LV}$ volumes were estimated by the Simpson method using apical two-chamber and four-chamber views, and LVEF was derived from these volumes in the standard manner. Left atrial volume was calculated by the method of disks using apical two-chamber and four-chamber views. LV volumes, LV mass and left atrial volume were indexed to body surface area. Peak septal mitral annular relaxation $\left(\mathrm{e}^{\prime}\right)$ velocity was assessed using tissue Doppler imaging. End-diastolic pressure (EDP) was calculated from septal $\mathrm{E} / \mathrm{e}^{\prime}$ as previously reported using the formula: $11.96+(0.596 \times$ septal E/e' $) .{ }^{15}$ Global longitudinal and circumferential strain were derived from speckle-tracking echocardiography.

\section{Exercise protocol}

Exercise tests were performed in the cardiopulmonary exercise laboratory at Brigham and Women's Hospital using ramp protocols, with the subjects breathing room air. ${ }^{12}$ All patients performed symptom-limited CPET, and pharmacological therapy was maintained prior to and through exercise testing. $\mathrm{VO}_{2}, \mathrm{VCO}_{2}$ and $\mathrm{VE}$ were measured breath by breath and averaged over a $10 \mathrm{~s}$ interval, using a gas analysis system (MGC Diagnostics, St. Paul, Minnesota, USA). Peak $\mathrm{VO}_{2}$ was estimated as the highest $10 \mathrm{~s}$ averaged $\mathrm{VO}_{2}$ during the last stage of the symptom-limited exercise test. Percent of predicted peak $\mathrm{VO}_{2}$ was determined by the Wasserman formula. ${ }^{16} \mathrm{VE} / \mathrm{VCO}_{2}$ slope was estimated from rest to the gas exchange at peak exercise.
Standard cuff sphygmomanometers were used to measure blood pressure, while CPET-associated electrocardiograms were used to obtain resting and peak heart rate. Age-predicted maximal heart rate was defined as 220 -age (years). ${ }^{17}$ Chronotropic index was estimated as (peak heart rate-resting heart rate)/(age-predicted maximal heart rate-resting heart rate). ${ }^{18}$

\section{Outcomes}

Clinical outcomes included all-cause death and the composite outcome of all-cause death, heart transplantation or LV assistant device (LVAD) implantation up to 31 December 2014. Heart transplantations and LVAD implantations were abstracted by chart review by researchers blinded to the CPET data. The National Death Index was used to determine all-cause death. Among patients with CTHF, cause of death was ascertained based on autopsy data $(\mathrm{n}=1)$ and, if no autopsy data were available $(n=16)$, the cause of death stated in the medical records by the treating physician was used.

\section{Statistical analysis}

Continuous variables are shown as mean \pm SD and median (25th, 75th percentiles) for normally and non-normally distributed data, respectively. Categorical variables are presented as number of subjects. Differences in clinical, CPET and echocardiographic characteristics between patients with CTHF and NCTHF were evaluated by unpaired t-test for normally distributed variables, Wilcoxon test for non-normally distributed variables and $\chi^{2}$ test for categorical variables. Differences in CPET variables between the studied groups were further adjusted for age, sex and body mass index, using multivariable regression analysis. Echocardiographic data are shown as multivariable adjusted means with $p$ values estimated from linear regression. The rates of incident outcomes are presented as events per 100 person-years at risk.

Univariate and multivariable Cox proportional-hazards regression models were used to assess the association between CTHF and clinical outcomes. Covariates for multivariable Cox regression models included age, sex and variables that associated with the composite outcome of all-cause death, heart transplantation or LVAD implantation using a forward stepwise selection procedure (retention $\mathrm{p}<0.10$ ) including all clinical, CPET and treatment variables assessed at baseline as candidate variables. Two multivariable Cox regression models were constructed: the first model included age and sex; and the second model further included LVEF, chronic kidney disease, resting heart rate, resting systolic blood pressure, pacemaker use, peak $\mathrm{VO}_{2}$ and $\mathrm{VE} / \mathrm{VCO}_{2}$ slope. We also performed sensitivity analyses repeating the primary analysis among the patients with CTHF stratified by the type of cancer treatment: (1) chemotherapy with anthracyclines only, (2) chest radiotherapy with anthracyclines and (3) chest radiotherapy only.

Statistical analysis was performed using Stata software V.13.1 (Stata Corp LP, College Station, Texas, USA). P values $<0.05$ were considered significant.

\section{RESULTS}

\section{Baseline characteristics}

Of the 969 individuals with HF included in the present study, 75 (8\%) had CTHF. Patients with CTHF were younger, more likely to be women and had lower body mass index and higher LVEF than patients with NCTHF (table 1). Compared with NCTHF, CTHF had lower prevalence of coronary artery disease, hypertension, diabetes mellitus and atrial fibrillation, higher prevalence of preserved (>50\%) and mid-range (40\%-49\%) LVEF, 


\begin{tabular}{|c|c|c|c|}
\hline Variables & NCTHF $(n=894)$ & CTHF $(n=75)$ & $P$ values \\
\hline Age, years & $55.6 \pm 13.6$ & $48.2 \pm 14.1$ & $<0.001$ \\
\hline Male, n (\%) & $628(70)$ & $26(35)$ & $<0.001$ \\
\hline White, n (\%) & $751(84)$ & $61(81)$ & 0.55 \\
\hline Body mass index, $\mathrm{kg} / \mathrm{m}^{2}$ & $28.8 \pm 6.1$ & $26.6 \pm 5.7$ & 0.003 \\
\hline NYHA, n (\%) & & & 0.50 \\
\hline 1 & $265(30)$ & $28(37)$ & \\
\hline ॥ & $312(35)$ & $22(29)$ & \\
\hline III & $265(30)$ & $22(29)$ & \\
\hline IV & $52(6)$ & $3(4)$ & \\
\hline Ischaemic cardiomyopathy, n (\%) & $224(25)$ & $4(5)$ & $<0.001$ \\
\hline Hypertension, $\mathrm{n}(\%)$ & $538(60)$ & $26(35)$ & $<0.001$ \\
\hline Diabetes mellitus, n (\%) & $239(27)$ & $11(15)$ & 0.022 \\
\hline Coronary artery disease, $\mathrm{n}(\%)$ & $325(36)$ & $17(23)$ & 0.017 \\
\hline Atrial fibrillation, $\mathrm{n}(\%)$ & $306(34)$ & $14(19)$ & 0.006 \\
\hline COPD, n (\%) & $88(10)$ & $5(7)$ & 0.37 \\
\hline Chronic kidney disease, n (\%) & $247(28)$ & $17(23)$ & 0.35 \\
\hline Anaemia, n (\%) & $233(26)$ & $18(24)$ & 0.70 \\
\hline LVEF, \% & $30[20,40]$ & $40[26,50]$ & $<0.001$ \\
\hline LVEF categories & & & 0.005 \\
\hline$<40 \%, \mathrm{n}(\%)$ & $594(66)$ & $36(48)$ & \\
\hline $40 \%-49 \%, n(\%)$ & $126(14)$ & $18(24)$ & \\
\hline$\geq 50 \%, \mathrm{n}(\%)$ & $174(20)$ & $21(28)$ & \\
\hline Recovered LVEF, n (\%) & $164(18)$ & $21(28)$ & 0.041 \\
\hline CRT/ICD, n (\%) & $391(44)$ & $16(21)$ & $<0.001$ \\
\hline Pacemaker, n (\%) & $411(46)$ & $20(27)$ & 0.001 \\
\hline Beta-blocker, n (\%) & 770 (86) & $52(69)$ & $<0.001$ \\
\hline ACEI/ARB, n (\%) & $710(79)$ & $53(71)$ & 0.08 \\
\hline Aldosterone antagonist, $\mathrm{n}(\%)$ & $259(29)$ & $21(28)$ & 0.86 \\
\hline Diuretic, n (\%) & $607(68)$ & $39(52)$ & 0.005 \\
\hline Calcium channel blocker, $\mathrm{n}(\%)$ & $70(8)$ & $5(7)$ & 0.72 \\
\hline Anticoagulation, $\mathrm{n}(\%)$ & $316(35)$ & $18(24)$ & 0.047 \\
\hline Antiplatelet, n (\%) & $477(53)$ & $19(25)$ & $<0.001$ \\
\hline Antiarrhythmic, n (\%) & $290(32)$ & $20(27)$ & 0.30 \\
\hline Statin, $n(\%)$ & $439(49)$ & $30(40)$ & 0.13 \\
\hline
\end{tabular}

Data are presented as mean \pm SD for normally distributed variables and median [25th, 75th percentiles] for non-normally distributed continuous variables.

ACEI/ARB, ACE inhibitor or angiotensin receptor blocker; COPD, chronic pulmonary obstructive disease; CTHF, cancer therapy-induced heart failure; CRT/ICD, cardiac resynchronisation therapy and/or implantable cardioverter defibrillator; LVEF, left ventricular ejection fraction; NCTHF, non-cancer therapy-induced heart failure; NYHA, New York Heart Association Classification.

and higher prevalence of recovered LVEF. Patients with CTHF were less likely to be on beta-blockers and diuretics, or to have pacemakers, implantable cardioverter defibrillators and/or cardiac resynchronisation therapy than NCTHF.

In analyses adjusted for age and sex, compared with NCTHF, CTHF had higher LVEF, but similar global longitudinal and circumferential strains (table 2). CTHF was also associated with lower LV and left atrial volumes and LV mass, higher E/A ratio and $\mathrm{E} / \mathrm{e}^{\prime}$ ratio consistent with higher $\mathrm{LV}$ filling pressure, and higher EDP/left atrial volume index ratio and EDP/LV end-diastolic volume index ratio, indicating lower compliance of left-sided cardiac chambers (table 2 and online supplemental figure 1). Accounting for between-group differences in age, sex and LVEF, patients with CTHF further demonstrated lower $\mathrm{e}^{\prime}$ indicating worse LV relaxation, and higher global longitudinal strain, indicating worse contractile function. No differences in right ventricular size or fractional area change were observed between the groups (data not shown).
Table 2 Adjusted baseline echocardiographic characteristics of patients with CTHF and NCTHF with available echocardiographic images

\begin{tabular}{|c|c|c|c|}
\hline Variables & NCTHF $(n=834)^{*}$ & CTHF $(n=71)^{*}$ & $P$ values* \\
\hline LVEF, \% & $32(31,32)$ & $36(33,40)$ & 0.048 \\
\hline LVEDVI, $\mathrm{mL} / \mathrm{m}^{2}$ & $92.7 \pm 1.4$ & $75.4 \pm 4.9$ & $<0.001$ \\
\hline LVESVI, $\mathrm{mL} / \mathrm{m}^{2}$ & $63.6 \pm 1.3$ & $48.5 \pm 4.7$ & 0.002 \\
\hline Mean wall thickness, cm & $0.98 \pm 0.01$ & $0.94 \pm 0.03$ & 0.16 \\
\hline LV mass index, $\mathrm{g} / \mathrm{m}^{2}$ & $117.3 \pm 1.3$ & $96.7 \pm 4.8$ & $<0.001$ \\
\hline Relative wall thickness & $0.37 \pm 0.004$ & $0.38 \pm 0.02$ & 0.57 \\
\hline E/A ratio & $1.59 \pm 0.04$ & $1.90 \pm 0.15$ & 0.042 \\
\hline $\mathrm{e}^{\prime}$ septal, cm/s & $6.3 \pm 0.1$ & $5.8 \pm 0.3$ & 0.12 \\
\hline E/e' septal & $14.5 \pm 0.3$ & $17.0 \pm 1.1$ & 0.026 \\
\hline $\begin{array}{l}\text { EDP/LVEDVI ratio, } \mathrm{mm} \mathrm{Hg} / \\
\mathrm{mL} / \mathrm{m}^{2}\end{array}$ & $0.26 \pm 0.004$ & $0.33 \pm 0.01$ & $<0.001$ \\
\hline $\mathrm{LAVI}, \mathrm{mL} / \mathrm{m}^{2}$ & $37.7 \pm 0.6$ & $30.2 \pm 2.2$ & 0.001 \\
\hline $\begin{array}{l}\text { EDP/LAVI ratio, } \mathrm{mm} \mathrm{Hg} / \\
\mathrm{mL} / \mathrm{m}^{2}\end{array}$ & $0.66 \pm 0.01$ & $0.88 \pm 0.04$ & $<0.001$ \\
\hline $\begin{array}{l}\text { Global longitudinal LV } \\
\text { strain, \% }\end{array}$ & $-12.6 \pm 0.2$ & $-12.5 \pm 0.6$ & 0.89 \\
\hline $\begin{array}{l}\text { Global circumferential LV } \\
\text { strain, \% }\end{array}$ & $-18.9 \pm 0.3$ & $-19.6 \pm 0.8$ & 0.41 \\
\hline \multicolumn{4}{|c|}{$\begin{array}{l}\text { Data are presented as mean } \pm \text { SE for all variables, except LVEF, which is presented as } \\
\text { geometric mean }(95 \% \mathrm{CI}) \text {. The presented values of echocardiographic variables are } \\
\text { adjusted for age and sex. } \\
\text { *Adjusted for age and sex. } \\
\text { CTHF, cancer therapy-induced heart failure; EDP, end-diastolic pressure; LAVI, left } \\
\text { atrial volume index; LV, left ventricular; LVEDVI, left ventricular end-diastolic volume } \\
\text { index; LVEF, left ventricular ejection fraction; LVESVI, left ventricular end-systolic } \\
\text { volume index; NCTHF, non-cancer therapy-induced heart failure. }\end{array}$} \\
\hline
\end{tabular}

On cardiopulmonary exercise testing, the mean peak respiratory exchange ratio was $>1.1$ in both the CTHF and NCTHF groups, consistent with adequate and comparable exercise effort between groups (table 3). Functional capacity, measured as the peak $\mathrm{VO}_{2}$ (absolute and percent of predicted), was similarly impaired between CTHF and NCTHF, while ventilatory efficiency based on the $\mathrm{VE} / \mathrm{VCO}_{2}$ slope was similarly abnormal (elevated). In contrast, CTHF had higher resting and peak heart rate, and chronotropic index, but lower resting systolic and diastolic blood pressure and peak systolic blood pressure as compared with NCTHF. These results did not change after adjusting for age, sex and body mass index, except for the differences in blood pressure measurements, which became non-significant (table 3). In addition, further adjustment for beta-blocker use did not appreciably change the differences in heart rate-derived variables between the studied groups (data not shown).

\section{Outcomes}

During a median follow-up of $4.5(3.0-5.8)$ years, there were 187 (19\%; 170 in NCTHF, 17 in CTHF) deaths, 62 (6\%; 58 in NCTHF, 4 in CTHF) LVAD implantations, 55 (6\%; 52 in NCTHF, 3 in CTHF) transplants and $256(26 \% ; 235$ in NCTHF, 21 in CTHF) composite events. Among the 17 patients with CTHF who died, the causes of death were cancer $(n=2)$, end-stage HF $(n=6)$ and stroke, myocardial infarction or sudden death $(n=4)$. In five patients, cause of death could not be definitively assessed, although all had symptomatic HF (NYHA class II-III) at the time of death. In unadjusted analysis, CTHF and NCTHF had similar rates of death and of the composite endpoint (table 4). However, after adjustment for age and sex, CTHF was associated with a higher risk of death $(p=0.003)$ and a marginally higher risk of the composite endpoint $(\mathrm{p}=0.057)$ (figure 1 and table 4). 
Table 3 Baseline cardiopulmonary exercise testing characteristics of the participants.

\begin{tabular}{lcccc}
\hline Variables & $\begin{array}{l}\text { NCTHF } \\
(\mathbf{n}=894)\end{array}$ & $\begin{array}{l}\text { CTHF } \\
(\mathbf{n}=75)\end{array}$ & P values* & P valuest \\
\hline Peak VO $_{2^{\prime}} \mathrm{mL} / \mathrm{min} / \mathrm{kg}$ & $15.3 \pm 6.3$ & $16.0 \pm 5.8$ & 0.33 & 0.40 \\
\hline$\%$ Predicted peak VO $_{2}$ & $61.2 \pm 20.2$ & $62.4 \pm 20.2$ & 0.62 & 0.44 \\
\hline VE/VCO $_{2}$ slope & $33.0 \pm 8.8$ & $32.4 \pm 6.9$ & 0.55 & 0.43 \\
\hline Haemodynamic & & & & \\
\hline Resting HR, bpm & $72 \pm 14$ & $79 \pm 16$ & $<0.001$ & $<0.001$ \\
\hline Peak HR, bpm & $122 \pm 28$ & $138 \pm 31$ & $<0.001$ & 0.003 \\
\hline Chronotropic index & $0.53 \pm 0.28$ & $0.64 \pm 0.30$ & 0.001 & 0.024 \\
\hline HR recovery at 1 min, bpm & $20 \pm 15$ & $22 \pm 17$ & 0.25 & 0.11 \\
\hline Resting SBP, mm Hg & $117 \pm 20$ & $112 \pm 19$ & 0.020 & 0.64 \\
\hline Peak SBP, mm Hg & $141 \pm 29$ & $134 \pm 29$ & 0.049 & 0.29 \\
\hline Resting DBP, mm Hg & $74 \pm 11$ & $71 \pm 13$ & 0.036 & 0.46 \\
\hline Peak DBP, mm Hg & $75 \pm 12$ & $73 \pm 13$ & 0.18 & 0.46 \\
\hline Peak RER & $1.19 \pm 0.13$ & $1.23 \pm 0.14$ & 0.006 & 0.048 \\
\hline
\end{tabular}

Data are presented as mean \pm SD.

*Unadjusted.

†Adjusted for age, sex and body mass index.

CTHF, cancer therapy-induced heart failure; DBP, diastolic blood pressure; HR, heart rate; NCTHF, non-cancer therapy-induced heart failure; RER, respiratory exchange ratio; SBP, systolic blood pressure.

In multivariable Cox regression analysis adjusted for age, sex, LVEF, chronic kidney disease, resting heart rate, resting systolic blood pressure, pacemaker use, peak $\mathrm{VO}_{2}$ and $\mathrm{VE} / \mathrm{VCO}_{2}$ slope, CTHF was associated with a significantly higher risk of death (HR 2.64; 95\% CI 1.53 to $4.55 ; \mathrm{p}=0.001$ ) and the composite endpoint (HR 1.79; 95\% CI 1.10 to $2.91 ; \mathrm{p}=0.019$ ) (table 4). Further adjustment for ACE inhibitors/angiotensin receptor blockers and beta-blocker use, and chronotropic index did not significantly change these findings (online supplemental table 1).

\section{Relationship of echocardiographic variables with cardiopulmonary response to exercise and outcomes}

In analyses adjusted for age and sex, lower LVEF, lower $\mathrm{e}^{\prime}$ and higher $\mathrm{E} / \mathrm{e}^{\prime}$ were each associated with lower peak $\mathrm{VO}_{2}$, higher $\mathrm{VE} / \mathrm{VCO}_{2}$ slope and higher incidence of the composite endpoint in both CTHF and NTCHF (table 5). Higher EDP/LV end-diastolic volume index and EDP/left atrial volume index ratios were associated with a higher risk of the composite endpoint in both CTHF and NCTHF . Higher global longitudinal strain was associated with lower peak $\mathrm{VO}_{2}$ and higher $\mathrm{VE} / \mathrm{VCO}_{2}$ slope in both CTHF and NCTHF groups and with the composite endpoint in
NCTHF, and showed a similar although non-significant trend towards worse outcomes in CTHF (table 5).

\section{Impact of cancer treatment type}

Among the patients with CTHF, 42 were exclusively treated with anthracyclines, 25 underwent chest radiotherapy with adjuvant anthracycline chemotherapy and 8 exclusively underwent chest radiotherapy. These subgroups had similar baseline characteristics, echocardiographic features and CPET parameters, except for a higher prevalence of coronary artery disease and ischaemic cardiomyopathy, lower prevalence of chronic kidney disease and lower heart rate recovery after termination of CPET in patients who exclusively underwent radiotherapy (online supplemental tables 2-4). Furthermore, the subgroup that exclusively underwent radiotherapy had higher risk of death compared with the two other subgroups in fully adjusted analysis (online supplemental table 5).

\section{DISCUSSION}

This study's comprehensive comparison of the cardiovascular profile of patients with CTHF and NCTHF provides three major novel findings. First, despite being younger with a lower prevalence of cardiovascular comorbidities, CTHF had similar impairment in functional capacity and ventilatory efficiency as NCTHF. Second, CTHF was characterised by higher LVEF, but had similar global longitudinal strain and worse LV diastolic properties compared with NCTHF, and worse LV diastolic function predicted adverse outcomes in both groups. Third, although CTHF and NCTHF had similar rates of death and the composite endpoint in unadjusted analysis, after accounting for differences in clinical profile and LVEF, CTHF was associated with a significantly higher risk of these adverse outcomes. Together, these findings demonstrate that CTHF represents a HF subgroup with distinct clinical and echocardiographic characteristics and a worse prognosis, and suggest that impairments in diastolic function may be particularly relevant in long-term cancer survivors who develop HF.

Prominent differences in clinical characteristics were noted between the CTHF and NCTHF groups. Consistent with prior studies in HF, ${ }^{67911}$ patients with CTHF were more likely to be women. Potential explanations for this finding include a higher proportion of breast cancer and the fact that women treated with anthracyclines are more likely to develop cardiac damage than men. ${ }^{19} 20$ We also confirmed that patients with CTHF were younger and had lower prevalence of cardiovascular

Table 4 Outcomes of study participants

\begin{tabular}{|c|c|c|c|c|c|c|c|c|}
\hline & $\begin{array}{l}\text { Number of } \\
\text { events/at risk }\end{array}$ & $\begin{array}{l}\text { Rate }(95 \% \mathrm{Cl}) \text { per } \\
100 \text { person-years }\end{array}$ & $\begin{array}{l}\text { HR }(95 \% \mathrm{Cl}) \\
\text { (unadjusted) }\end{array}$ & $P$ values & $\begin{array}{l}\text { HR }(95 \% \mathrm{Cl}) \\
\text { (adjusted*) }\end{array}$ & $P$ values & $\begin{array}{l}\text { HR }(95 \% \mathrm{Cl}) \\
\text { (adjustedt) }\end{array}$ & $P$ values \\
\hline \multicolumn{9}{|l|}{ Death $\ddagger$} \\
\hline NCTHF & $170 / 894$ & 4.4 (3.8 to 5.1$)$ & Ref & & Ref & & Ref & \\
\hline CTHF & $17 / 75$ & 5.2 (3.3 to 8.4$)$ & 1.21 (0.73 to 1.99$)$ & 0.46 & 2.24 (1.32 to 3.79$)$ & 0.003 & 2.64 (1.53 to 4.55$)$ & 0.001 \\
\hline \multicolumn{9}{|c|}{ Composite endpoint $\ddagger \S$} \\
\hline NCTHF & $235 / 894$ & 6.4 (5.7 to 7.3 ) & Ref & & Ref & & Ref & \\
\hline CTHF & $21 / 75$ & 6.7 (4.4 to 10.3$)$ & 1.05 (0.67 to 1.64$)$ & 0.84 & 1.57 (0.99 to 2.51$)$ & 0.057 & 1.79 (1.10 to 2.91$)$ & 0.019 \\
\hline
\end{tabular}

The composite endpoint was defined as the composite outcome of left ventricular assistant device (LVAD) implantation, heart transplantation or all-cause mortality.

${ }^{*}$ Adjusted for age and sex.

†Adjusted for age, sex, ejection fraction, chronic kidney disease, resting heart rate, resting systolic blood pressure, pacemaker use, peak VO 2 and VE/VCO relationship.

¥Median follow-up: CTHF group=4.7 (3.0 to 5.9) years; NCTHF group=4.4 (3.0 to 5.8) years.

$\S$ The composite endpoint included 4 LVAD, 3 transplants and 14 deaths in the CTHF groups; and 58 LVAD, 25 transplants and 152 deaths in the NCTHF group.

CTHF, cancer therapy-induced heart failure; NCTHF, non-cancer therapy-induced heart failure. 

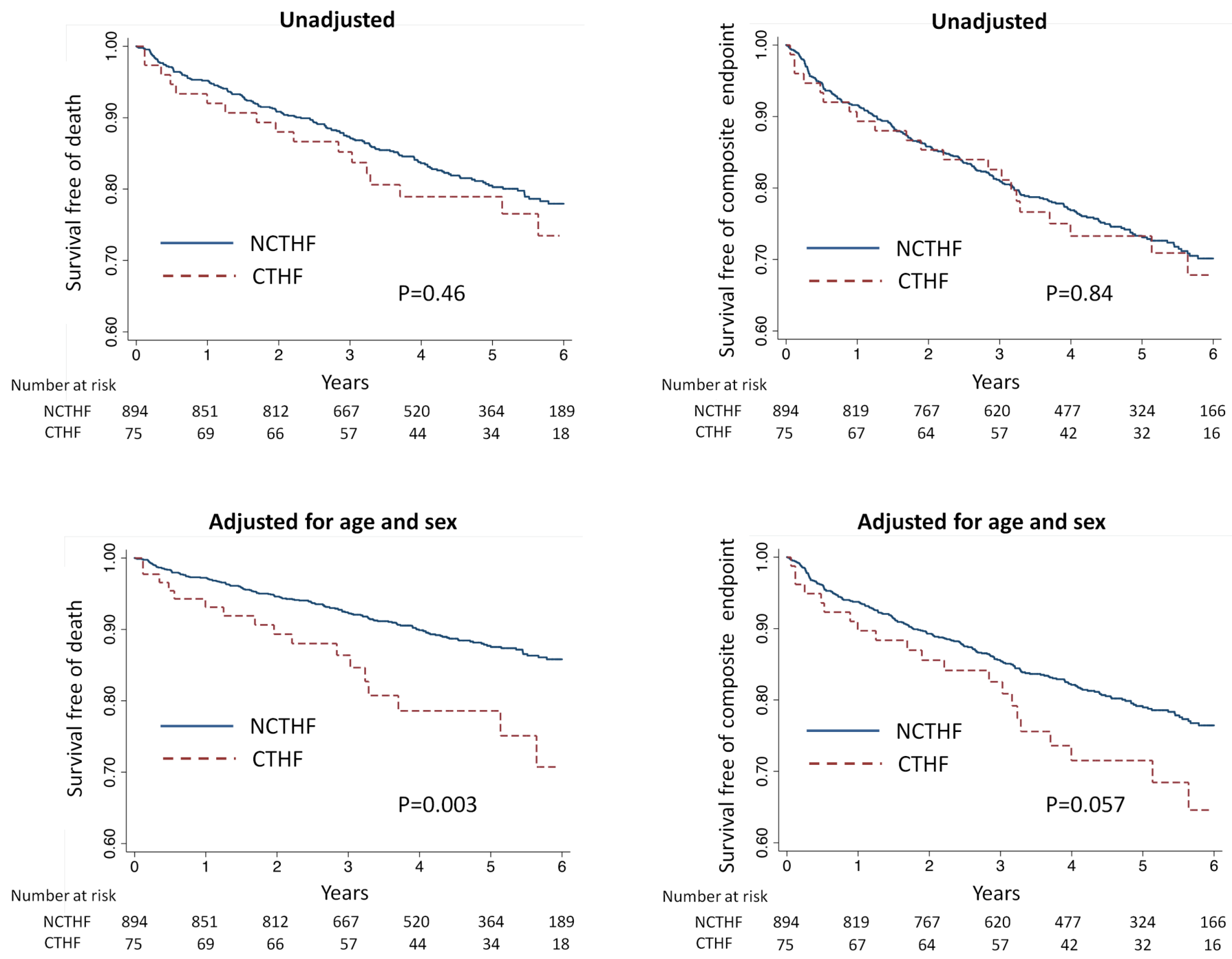

Figure 1 Kaplan-Meier curves for death and composite endpoint. The composite endpoint was defined as the composite outcome of left ventricular assistant device implantation, heart transplantation or all-cause mortality. CTHF, cancer therapy-induced heart failure; NCTHF, non-cancer therapyinduced heart failure.

comorbidities than NCTHF, ${ }^{67}$ including hypertension, diabetes, coronary artery disease and atrial fibrillation.

CTHF was associated with a similar risk of death when compared with NCTHF in unadjusted analysis. However, after accounting for differences in clinical characteristics including common cardiovascular and HF risk factors, CTHF was associated with significantly greater mortality than NCTHF. This finding agrees with data obtained in patients with HF recruited between 1982 and 1997, which showed a higher risk of death in 15 patients with anthracycline-induced HF when compared with 1215 patients with other causes of HF, after a mean of 4.4 years of follow-up. ${ }^{8}$ Importantly, our study-which included a larger number of cancer-treated patients-extends this finding to an era with substantially improved treatments for HF as compared with that prior report. ${ }^{8}$ While we cannot definitively exclude that active cancer contributed to the higher mortality among patients with CTHF, active cancer was the cause of death in only $12 \%$ of patients with CTHF in our study. Several recent reports have studied patients with severe HF receiving advanced therapies, such as heart transplant and/or LVAD implantation, and showed similar ${ }^{6910}$ or even lower ${ }^{11}$ risk of death in CTHF than NCTHF. Our study, in contrast, evaluates prognosis and outcomes up to LVAD implantation and/or transplantation. Our finding that the
CTHF group is at increased risk of adverse outcomes, coupled with their equivalent prognosis to NCTHF after LVAD implantation/transplantation, argues for careful monitoring of patients with CTHF despite frequently having a relatively preserved LVEF.

Despite the generally favourable demographic and clinical characteristics of CTHF relative to NCTHF, functional capacity quantified as peak $\mathrm{VO}_{2}$ and ventilatory efficiency quantified as the $\mathrm{VE} / \mathrm{VCO}_{2}$ slope were similar between groups. These findings suggest a similar degree of cardiac limitation in CTHF as NCTHF, despite a lower burden of cardiovascular comorbidities and favourable LVEF in CTHF. Concordantly, global longitudinal strain, a sensitive measure of LV systolic function, ${ }^{21}$ was similarly impaired in both CTHF and NCTHF groups after adjusting for age and sex. CTHF was also characterised by higher $\mathrm{LV}$ filling pressure (E/e' ratio, E/A ratio) and greater LV diastolic chamber stiffness (EDP/LV end-diastolic volume index ratio), all consistent with worse diastolic function and greater LV stiffness and in CTHF. Importantly, these are robust risk factors for adverse events in HF regardless of LVEF, ${ }^{22} 23$ and each measure was significantly associated with heightened risk of adverse outcomes in CTHF. These findings reinforce current recommendations and evidence that 
Table 5 Relationship between left ventricular measures assessed by echocardiography and CPET variables and outcomes adjusted for age and sex

\begin{tabular}{|c|c|c|c|c|}
\hline & \multicolumn{2}{|l|}{ NCTHF } & \multicolumn{2}{|l|}{ CTHF } \\
\hline & Beta \pm SE & $P$ values & Beta \pm SE & $P$ values \\
\hline \multicolumn{5}{|c|}{ Dependent variable: peak $\mathrm{VO}_{2}$} \\
\hline LVEF & $0.97 \pm 0.08$ & $<0.001$ & $0.89 \pm 0.25$ & 0.001 \\
\hline $\mathrm{e}^{\prime}$ septal & $0.09 \pm 0.01$ & $<0.001$ & $0.10 \pm 0.05$ & 0.053 \\
\hline E/e' septal & $-0.39 \pm 0.05$ & $<0.001$ & $-0.48 \pm 0.19$ & 0.016 \\
\hline EDP/LVEDVI ratio $\times 10$ & $0.00 \pm 0.07$ & 0.84 & $-0.06 \pm 0.04$ & 0.11 \\
\hline EDP/LAVI ratio $\times 10$ & $0.02 \pm 0.01$ & 0.10 & $-0.05 \pm 0.05$ & 0.32 \\
\hline $\begin{array}{l}\text { Global longitudinal LV } \\
\text { strain, \% }\end{array}$ & $-0.34 \pm 0.03$ & $<0.001$ & $-0.28 \pm 0.08$ & 0.001 \\
\hline \multicolumn{5}{|c|}{ Dependent variable: $\mathrm{VE} / \mathrm{VCO}_{2}$ slope } \\
\hline LVEF & $-0.61 \pm 0.06$ & $<0.001$ & $-0.73 \pm 0.23$ & 0.003 \\
\hline e' septal & $-0.06 \pm 0.01$ & $<0.001$ & $-0.09 \pm 0.05$ & 0.073 \\
\hline E/e' septal & $0.27 \pm 0.04$ & $<0.001$ & $0.43 \pm 0.20$ & 0.035 \\
\hline EDP/LVEDVI ratio $\times 10$ & $-0.01 \pm 0.01$ & 0.24 & $0.05 \pm 0.04$ & 0.19 \\
\hline EDP/LAVI ratio ×10 & $0.01 \pm 0.01$ & 0.99 & $-0.01 \pm 0.05$ & 0.77 \\
\hline \multirow{2}{*}{$\begin{array}{l}\text { Global longitudinal LV } \\
\text { strain, \% }\end{array}$} & $0.19 \pm 0.02$ & $<0.001$ & $0.23 \pm 0.09$ & 0.012 \\
\hline & HR $(95 \% \mathrm{Cl})$ & $P$ values & $\mathrm{HR}(95 \% \mathrm{Cl})$ & $P$ values \\
\hline \multicolumn{5}{|c|}{ Outcome: composite endpoint } \\
\hline LVEF & $\begin{array}{l}0.94 \\
(0.93 \text { to } 0.95)\end{array}$ & $<0.001$ & $\begin{array}{l}0.96 \\
(0.93 \text { to } 0.99)\end{array}$ & 0.028 \\
\hline$e^{\prime}$ septal & $\begin{array}{l}0.85 \\
\text { (0.78 to } 0.92)\end{array}$ & $<0.001$ & $\begin{array}{l}0.68 \\
(0.48 \text { to } 0.97)\end{array}$ & 0.034 \\
\hline E/e' septal & $\begin{array}{l}1.04 \\
\text { (1.02 to } 1.05)\end{array}$ & $<0.001$ & $\begin{array}{l}1.09 \\
\text { (1.02 to } 1.16)\end{array}$ & 0.012 \\
\hline EDP/LVEDVI ratio $\times 10$ & $\begin{array}{l}0.72 \\
\text { (0.59 to } 0.88)\end{array}$ & 0.001 & $\begin{array}{l}1.39 \\
(1.00 \text { to } 1.92)\end{array}$ & 0.047 \\
\hline EDP/LAVI ratio $\times 10$ & $\begin{array}{l}0.80 \\
\text { (0.69 to } 0.93)\end{array}$ & 0.004 & $\begin{array}{l}1.62 \\
\text { (1.16 to } 1.23)\end{array}$ & 0.004 \\
\hline $\begin{array}{l}\text { Global longitudinal LV } \\
\text { strain, \% }\end{array}$ & $\begin{array}{l}1.19 \\
\text { (1.13 to } 1.25)\end{array}$ & $<0.001$ & $\begin{array}{l}1.15 \\
(0.98 \text { to } 1.35)\end{array}$ & 0.09 \\
\hline
\end{tabular}

The composite endpoint was defined as the composite outcome of left ventricular assistant device implantation, heart transplantation or all-cause mortality.

CPET, cardiopulmonary exercise testing; CTHF, cancer therapy-induced heart failure; EDP, end-diastolic pressure; LAVI, left atrial volume index; LV, left ventricular; LVEF, left ventricular ejection fraction; LVEDVI, left ventricular end-diastolic volume index; NCTHF, non-cancer therapy-induced heart failure.

assessment of LV diastolic function should be systematically performed in individuals subjected to cancer therapy, ${ }^{24}{ }^{25}$ even though it remains uncertain whether improvements in LV diastolic function may be translated into better prognostic outcome in patients with $\mathrm{HF}^{26}$ We also noted higher EDP/left atrial volume index ratio in CTHF, consistent with lower left atrial compliance and suggesting cardiac stiffening beyond just the LV in CTHF. Although the impaired performance in CTHF is certainly multifactorial, with contributions from loss of skeletal muscle mass and radiotherapy-induced pulmonary disease among others, our findings highlight the importance of LV diastolic impairment in limiting functional capacity in CTHF. Smaller LV dimensions in CTHF compared with NCTHF have been previously reported, ${ }^{9}$ as has a higher prevalence of recovered LVEF. ${ }^{4}$ In contrast, similar or even worse LVEF values in CTHF compared with NCTHF have been previously reported by other groups. ${ }^{7910}$ The reasons for these differences in LVEF between studies are unclear, but likely relate to heterogeneity in the CTHF samples between studies including differences in the prevalence of recovered LVEF. Lastly, we observed that CTHF was associated with a higher resting heart rate, an established prognostic marker and treatment target in HF. ${ }^{27} 28$ While this finding suggests potentially worse autonomic function in CTHF, heart rate recovery was not worse in the CTHF group overall compared with NCTHF and the worse prognosis related to CTHF persisted after accounting for baseline heart rate in multivariable models.

Previous studies have demonstrated that anthracycline therapy and also chest radiotherapy increase the risk of HF. ${ }^{45}$ In the present report, we observed that patients exclusively treated with radiotherapy had higher rate of coronary artery disease and lower heart rate recovery after CPET termination as compared with patients exclusively treated with anthracyclines, concordant with previous studies showing that chest irradiation is a stimulus for coronary atherosclerosis ${ }^{29}$ and cardiac autonomic dysfunction. ${ }^{30}$ Although the number of patients and outcomes in each treatment history category was relatively small, subjects exclusively treated with radiotherapy had higher risk of death compared with subjects solely treated with anthracyclines and subjects treated with both anthracyclines and radiotherapy. Given the small numbers, further studies are necessary to confirm these findings.

Cancer therapy-induced cardiotoxicity has been classified into two categories: type 1 , traditionally seen with anthracyclines, which is frequently irreversible and dose related; and type 2 , classically seen with trastuzumab, primarily indicated for HER2-positive breast cancer, which is usually reversible (in 2-4 months after interruption) and not dose related. ${ }^{24}$ In our study, $25 \%$ of patients with CTHF had breast cancer and some of them possibly used trastuzumab. However, because the interval between cancer therapy and cardiorespiratory phenotyping in patients with CTHF was very long (median $=18$ years), we believe that the use of trastuzumab did not substantially influence cardiac measures. The long time interval between cancer therapy and evaluation of cardiorespiratory characteristics also indicates that most of the patients with CTHF were long-term cancer survivors, presenting late following cancer therapy. This is noteworthy because long-term cancer survivors with HF may have different management and outcomes than patients who developed HF during or early after anthracycline chemotherapy. For instance, patients with HF with a recent history of cancer might receive less aggressive treatment compared with other patients with HF. In our sample, the number of LVAD implantations or heart transplantations was similar in both HF groups $(n=110,12 \%$ of NCTHF; $n=7$, $9 \%$ of CTHF; $p=0.45$ ), supporting the notion that the use of advanced HF therapies did not differ in long-term cancer survivors and NCTHF.

Several limitations of this report should be acknowledged. First, this is an observational study and unmeasured confounding variables may have influenced the observed associations. Second, our sample consisted mainly of patients with advanced HF at a tertiary medical centre who were referred for CPET and therefore may not be representative of the general HF population or of CTHF specifically, thus potentially limiting the generalisability of our findings. Third, data on heart transplantation, LVAD implantation and HF hospitalisation were gathered by review of Brigham and Women's Hospital charts, which might have led to underestimation of these events. However, the rate of these outcomes occurring at an institution different from where the patient is being longitudinally followed is generally low. Fourth, time of cancer diagnosis and therapies were retrospectively ascertained, which might have introduced misclassifications. Fifth, 
data on natriuretic peptides, which are prognostically relevant biomarkers in HF, and on non-ischaemic HF aetiologies were not available in our study population. Sixth, in a subset of patients with CTHF who died (29\%), the cause of death could not be definitively ascertained.

\section{CONCLUSION}

Compared with NCTHF, patients with CTHF were younger, with fewer comorbidities and higher LVEF. Despite a more favourable clinical profile, extent of impairment in global longitudinal strain, functional capacity and ventilatory efficiency was similar between groups. Furthermore, LV diastolic function was worse in CTHF and was significantly associated with adverse outcomes. Accounting for differences in clinical characteristics, CTHF was associated with higher risk of all-cause mortality and the composite endpoint of all-cause death, heart transplantation or LVAD implantation. Impaired LV diastolic function may be particularly important in long-term cancer survivors who develop HF. Future studies are necessary to define the role of LV diastolic dysfunction in surveillance for long-term sequelae of cardiotoxic cancer therapy.

\section{Key messages}

\section{What is already known on this subject?}

- Among patients with heart failure (HF), there is limited information regarding the phenotype and prognosis of $\mathrm{HF}$ induced by cancer therapy (CTHF).

\section{What might this study add?}

- CTHF is characterised by a distinct clinical profile, better ejection fraction but worse left ventricular diastolic properties, and similarly impaired global longitudinal strain, functional capacity and ventilatory efficiency than $\mathrm{HF}$ not induced by cancer therapy (NCTHF). Worse left ventricular diastolic function predicted adverse outcomes in both groups. Accounting for differences in clinical characteristics, CTHF was associated with worse long-term prognosis than NCTHF.

\section{How might this impact on clinical practice?}

- Our results suggest that impairments in left ventricular diastolic function are particularly important in patients with CTHF, and that surveillance of both systolic and diastolic function is important to optimally detect the long-term sequelae of cardiotoxic cancer therapy.

Contributors WNJ and AMS contributed to the design of the work, and to the analysis and interpretation of data. EW, MoS, GLG, MaS, JDG, DEF, BC, HS and AN contributed to the acquisition, analysis and/or interpretation of data. WNJ drafted the work, and EW, MoS, GLG, MaS, JDG, DEF, BC, HS, AN and AMS revised it critically for important intellectual content. All authors approved the version to be published and are accountable for all aspects of the work in ensuring that questions related to the accuracy or integrity of any part of the work are appropriately investigated and resolved.

Funding This work was supported by NHLBI (K08HL116792 and R01HL135008 to AMS), AHA (14CRP20380422 to AMS), a Watkins Discovery Award from the Brigham and Women's Heart and Vascular Center to AMS and the Brazilian National Council for Scientific and Technological Development (306154/2017-0 to WNJ).

Competing interests AMS reports receiving research support from Novartis and Bellerophron, and consulting fees from Philips Ultrasound. The other authors have nothing to disclose.

Patient consent Not required.

Ethics approval Partners Human Research Committee.

Provenance and peer review Not commissioned; externally peer reviewed. (c) Article author(s) (or their employer(s) unless otherwise stated in the text of the article) 2019. All rights reserved. No commercial use is permitted unless otherwise expressly granted.

\section{REFERENCES}

1 Zamorano JL, Lancellotti P, Rodriguez Muñoz D, et al. ESC Position Paper on cancer treatments and cardiovascular toxicity developed under the auspices of the ESC Committee for Practice Guidelines: The Task Force for Cancer Treatments and Cardiovascular Toxicity of the European Society of Cardiology (ESC). Eur Heart J 2016:2016:2768-801.

2 Oliveira GH, Qattan MY, Al-Kindi S, et al. Advanced heart failure therapies for patients with chemotherapy-induced cardiomyopathy. Circ Heart Fail 2014;7:1050-8.

3 Yeh ET, Bickford CL. Cardiovascular complications of cancer therapy: incidence, pathogenesis, diagnosis, and management. J Am Coll Cardiol 2009:53:2231-47.

4 Mele D, Nardozza M, Spallarossa P, et al. Current views on anthracycline cardiotoxicity. Heart Fail Rev 2016;21:621-34.

5 Nolan MT, Russell DJ, Negishi K, et al. Meta-analysis of association between mediastinal radiotherapy and long-term heart failure. Am J Cardiol 2016;118:1685-91.

6 Oliveira GH, Hardaway BW, Kucheryavaya AY, et al. Characteristics and survival of patients with chemotherapy-induced cardiomyopathy undergoing heart transplantation. J Heart Lung Transplant 2012;31:805-10.

7 Shore S, Grau-Sepulveda MV, Bhatt DL, et al. Characteristics, treatments, and outcomes of hospitalized heart failure patients stratified by etiologies of cardiomyopathy. JACC Heart Fail 2015:3:906-16.

8 Felker GM, Thompson RE, Hare JM, et al. Underlying causes and long-term survival in patients with initially unexplained cardiomyopathy. N Engl J Med 2000;342:1077-84.

9 Oliveira GH, Dupont M, Naftel D, et al. Increased need for right ventricular support in patients with chemotherapy-induced cardiomyopathy undergoing mechanical circulatory support: outcomes from the INTERMACS Registry (Interagency Registry for Mechanically Assisted Circulatory Support). J Am Coll Cardiol 2014;63:240-8.

10 Thomas GR, McDonald MA, Day J, et al. A matched cohort study of patients with endstage heart failure from anthracycline-induced cardiomyopathy requiring advanced cardiac support. Am J Cardio/ 2016;118:1539-44.

11 Lenneman AJ, Wang L, Wigger M, et al. Heart transplant survival outcomes for adriamycin-dilated cardiomyopathy. Am J Cardiol 2013;111:609-12.

12 Nadruz W, West E, Santos M, et al. Heart failure and midrange ejection fraction: implications of recovered ejection fraction for exercise tolerance and outcomes. Circ Heart Fail 2016;9:e002826.

13 Levey AS, Stevens LA, Schmid CH, et al. A new equation to estimate glomerular filtration rate. Ann Intern Med 2009;150:604-12.

14 Shah AM, Cheng S, Skali H, et al. Rationale and design of a multicenter echocardiographic study to assess the relationship between cardiac structure and function and heart failure risk in a biracial cohort of community-dwelling elderly persons: the Atherosclerosis Risk in Communities study. Circ Cardiovasc Imaging 2014; 7:173-81.

15 Ommen SR, Nishimura RA, Appleton CP, et al. Clinical utility of Doppler echocardiography and tissue Doppler imaging in the estimation of left ventricular filling pressures: a comparative simultaneous Doppler-catheterization study. Circulation 2000;102:1788-94.

16 Hansen JE, Sue DY, Wasserman K. Predicted values for clinical exercise testing. Am Rev Respir Dis 1984;129:S49-S55.

17 Astrand I. Aerobic work capacity in men and women with special reference to age. Acta Physiol Scand Supp/ 1960;49:1-92.

18 Brubaker PH, Kitzman DW. Chronotropic incompetence: causes, consequences, and management. Circulation 2011;123:1010-20.

19 Lipshultz SE, Lipsitz SR, Mone SM, et al. Female sex and higher drug dose as risk factors for late cardiotoxic effects of doxorubicin therapy for childhood cancer. $N$ Engl J Med 1995;332:1738-44

20 Sorensen K, Levitt G, Sebag-Montefiore D, et al. Cardiac function in Wilms' tumor survivors. J Clin Oncol 1995;13:1546-56.

21 Shah AM, Claggett B, Sweitzer NK, et al. Prognostic importance of impaired systolic function in heart failure with preserved ejection fraction and the impact of spironolactone. Circulation 2015;132:402-14.

22 Burke MA, Katz DH, Beussink L, et al. Prognostic importance of pathophysiologic markers in patients with heart failure and preserved ejection fraction. Circ Heart Fail 2014; 7:288-99.

23 Shah AM, Claggett B, Sweitzer NK, et al. Cardiac structure and function and prognosis in heart failure with preserved ejection fraction: findings from the echocardiographic study of the Treatment of Preserved Cardiac Function Heart Failure with an Aldosterone Antagonist (TOPCAT) Trial. Circ Heart Fail 2014; 7:740-51.

24 Plana JC, Galderisi M, Barac A, et al. Expert consensus for multimodality imaging evaluation of adult patients during and after cancer therapy: a report from the American Society of Echocardiography and the European Association of Cardiovascular Imaging. J Am Soc Echocardiogr 2014;27:911-39. 
25 Armstrong GT, Joshi VM, Ness KK, et al. Comprehensive echocardiographic detection of treatment-related cardiac dysfunction in adult survivors of childhood cancer: results from the St. Jude Lifetime Cohort Study. J Am Coll Cardiol 2015;65:2511-22.

26 Nadruz W, Shah AM, Solomon SD. Diastolic dysfunction and hypertension. Med Clin North Am 2017;101:7-17.

27 Castagno D, Skali $\mathrm{H}$, Takeuchi $\mathrm{M}$, et al. Association of heart rate and outcomes in a broad spectrum of patients with chronic heart failure: results from the CHARM (Candesartan in Heart Failure: Assessment of Reduction in Mortality and morbidity) program. J Am Coll Cardiol 2012;59:1785-95.
28 Dobre D, Zannad F, Keteyian SJ, et al. Association between resting heart rate, chronotropic index, and long-term outcomes in patients with heart failure receiving $\beta$-blocker therapy: data from the HF-ACTION trial. Eur Heart J 2013;34:2271-80.

29 Darby SC, Ewertz M, McGale P, et al. Risk of ischemic heart disease in women after radiotherapy for breast cancer. N Engl J Med 2013;368:987-98.

30 Groarke JD, Tanguturi VK, Hainer J, et al. Abnormal exercise response in longterm survivors of Hodgkin lymphoma treated with thoracic irradiation: evidence of cardiac autonomic dysfunction and impact on outcomes. J Am Coll Cardiol 2015;65:573-83. 